\title{
Primera Evidencia Serológica de Infección por Hantavirus en Roedores, en Colombia
}

\author{
Ader Alemán ${ }^{1}$, Haydeé Iguarán², Henry Puerta ${ }^{3}$, César Cantillo ${ }^{4}$, James Mills ${ }^{5}$, William \\ Ariz $^{6}$ y Salim Mattar. ${ }^{7}$ \\ ${ }^{1}$ Médico Veterinario y Zootecnista. Instituto de Investigaciones Biológicas del Trópico, Facultad de Me- \\ dicina Veterinaria y Zootecnia. Universidad de Córdoba-Colombia. E-mail: Aderlaing@yahoo.com \\ ${ }^{2}$ Médico Veterinario y Zootecnista. Instituto de Investigaciones Biológicas del Trópico, Facultad de Me- \\ dicina Veterinaria y Zootecnia. Universidad de Córdoba, Colombia. E-mail: haidiguaran@yahoo.es \\ ${ }^{3}$ Bacteriólogo. Instituto de Investigaciones Biológicas del Trópico, Facultad de Medicina Veterinaria y \\ Zootecnia. Universidad de Córdoba-Colombia. E-mail: henry.puerta@gmail.com \\ ${ }^{4}$ Bacteriólogo. Instituto de Investigaciones Biológicas del Trópico, Facultad de Medicina Veterinaria y \\ Zootecnia. Universidad de Córdoba-Colombia. E-mail: cesar.ct@gmail.com \\ ${ }^{5}$ Ph.D. Chief, Medical Ecology Unit, Special Pathogens Branch, Division of Viral and Rickettsial Dis- \\ eases, National Center for Infectious Diseases, Centers for Disease Control and Prevention, 1600 Clifton \\ Road, Mail Stop G14, Atlanta, GA 30333, USA. E-mail: jum0@cdc.gov \\ ${ }^{6}$ Médico Veterinario y Zootecnista. Instituto de Investigaciones Biológicas del Trópico, Facultad de Me- \\ dicina Veterinaria y Zootecnia. Universidad de Córdoba-Colombia. \\ E-mail: wariz1278@yahoo.es \\ ${ }^{7}$ Ph.D. Director Instituto de Investigaciones Biológicas del Trópico, Facultad de Medicina Veterinaria y \\ Zootecnia. Universidad de Córdoba. E-mail: mattarsalim@hotmail.com
}

Recibido 28 Octubre 2005/Enviado para Modificación 20 Marzo 2006/Aceptado 19 Abril 2006

\section{RESUMEN}

Objetivo Determinar la prevalencia de infección por hantavirus en roedores del Departamento de Córdoba, Colombia.

Metodología Captura de roedores con trampas tipo Sherman live-capture

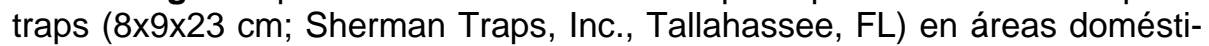
cas y peridomésticas en el departamento de Córdoba. Analisis de anticuerpos IgG por ELISA, empleando como antígeno una proteína recombinante de la nucleocapside del Sin Nombre Virus (SNV) (CDC, Atlanta, Georgia, USA).

Resultados Durante los meses de enero de 2003 a noviembre de 2004, en 79 noches de trampeo fueron capturados 336 roedores en once municipios del departamento de Córdoba (Murinae: 249; Sigmodontinae: 68; Heteromyidae: 17; Echimyidae: 2) (éxito de captura del 8,5\%). La seroprevalencia de anticuerpos contra hantavirus fue del 2,1 \% (7 de 336 capturas). Los porcentajes de seropositividad específicos por género oscilaron entre 5,9\% (1 de 17, Heteromys) a $50 \%$ (1 de 2, Proechimys). 
Conclusiones La prevalencia de anticuerpos contra el SNV en roedores de Córdoba, Colombia; indica que al menos un hantavirus es endémico en roedores del norte colombiano y esta frecuentemente trasmitido a residentes rurales.

Palabras Clave: Hantavirus, roedores, Colombia, seroprevalencia (fuente: DeCS, BIREME).

\section{ABSTRACT \\ First serological evidence of Hantavirus infection in rodents in Colombia}

Objective Determining Hantavirus infection prevalence in rodents in the Córdoba department, Colombia.

Methods Rodents were captured using Sherman live-capture traps $(8 \times 9 \times 23$ $\mathrm{cm}$; Sherman Traps, Inc., Tallahassee, FL) in peridomestic areas of Córdoba. Hantavirus IgG antibodies were detected by ELISA using Sin Nombre virus (SNV) recombinant nucleocapsid antigen (CDC, Atlanta, Georgia, USA).

Results 336 rodents were captured in 11 townships in the Córdoba department (Murinae: 249; Sigmodontinae: 68; Heteromyidae: 17; Echimyidae: 2; $8,5 \%$ overall trap success) during 79 nights of trapping between January 2003 and November 2004. Hantavirus antibody seroprevalence was 2,1 \% (7 out of 336 captures). Prevalence by genus varied between 5,9\% (1 out of 17 Heteromys) to $50 \%$ (1 out of 2 Proechimys).

Conclusions SNV-reactive antibody prevalence in rodents in Córdoba, Colombia, indicated that at least one hantavirus is endemic in rodents in northern Colombia and is frequently transmitted to rural residents.

Key Words: Hantavirus, rodent, Colombia, seroprevalence (source: MeSH, $N L M)$.

L

os hantavirus son un grupo de patógenos emergentes identificados en 1993 en los Estados Unidos como agentes etiológicos de una enfermedad zoonótica en las Américas denominada síndrome pulmonar por hantavirus (SPH) (1). Desde entonces, estos patógenos se han diseminado por todo el continente, asociados a roedores de la subfamilia Sigmodontinae y Arvicolinae (roedores del nuevo mundo). Sin embargo, el SPH solo ha sido notificado en países de centro y sur América como Panamá, Venezuela, Brasil, Bolivia, Uruguay, Paraguay, Argentina y Chile, con una mortalidad aproximada del $40 \%(2)$.

Estudios serológicos en roedores han permitido la identificación de reservorios putativos de hantavirus en Uruguay, Brasil y Argentina, y detec- 
tado anticuerpos en algunas especies de roedores en Paraguay, Venezuela y Panamá (3).

Colombia limita con países en los que se ha identificado y caracterizado hantavirus a partir de roedores reservorios y casos clínicos compatibles con $\mathrm{SPH}$. Muchas de estas regiones poseen condiciones geoclimáticas similares a la nuestra. La presencia de esta enfermedad, aún no ha sido clínicamente identificada en este país. Sin embargo, un estudio realizado en el departamento de Córdoba en el año 2004 logró evidenciar la circulación de anticuerpos contra hantavirus en una población de trabajadores del campo, mostrando una seroprevalencia del $13,5 \%$ con diferencias significativas entre grupos de diferentes edades (4). Antecedentes bibliográficos de distribución geográfica de roedores en el neotrópico, demuestran que los reservorios principales de hantavirus identificados en estos países de sur América circulan en el territorio colombiano (5). Ante la carencia de estudios sobre la detección de anticuerpos contra hantavirus en roedores de Colombia, se diseñó este trabajo con el objetivo de determinar la prevalencia de infección por hantavirus en roedores del departamento de Córdoba, Colombia.

\section{MATERIAL Y MÉTODOS}

Tipo de estudio, tiempo y cálculo del tamaño de la muestra

Se llevó a cabo un estudio prospectivo descriptivo de corte transversal durante los meses de Enero de 2003 a Noviembre de 2004. El cálculo del tamaño de la muestra fue realizado mediante el uso de la herramienta STATS ${ }^{\circledR}$ a partir de una población indeterminada de roedores, con una seroprevalencia esperada del 2 \% debido a estudios previos en Panamá con condiciones geográficas similares a las nuestras (6). Por lo tanto, asumiendo un nivel de significación del 99,9 \% y admitiendo un error del $5 \%$ se determinó capturar 266 roedores.

Áreas de estudio

Se estudiaron diferentes zonas urbanas y rurales ubicadas en los municipios de Lorica (LOR), Planeta Rica (PR), Montería (MON), Ayapel (AYA), Cereté (CER), Sahagún (SAH), Buena vista (BTA), Purísima (PUR), Cienaga de oro (CO), Pueblo Nuevo (PVO) y San Pelayo (PYO) en el Departamento de Córdoba-Colombia (Figura 1).

Para la captura de los roedores, se realizó un trampeo intensivo haciendo especial énfasis en zonas internas y aledañas a las áreas donde la prevalencia 
de infección por hantavirus había sido previamente descrita por Máttar et al. (4). Las áreas de estudio fueron clasificadas como areas domésticas y peridomésticas conformadas por todos los sitios internos y aledaños a viviendas urbanas y rurales, como son: jardines, caminos, vías de acceso, sitios cercados, cultivos, zonas verdes, casas de fincas, construcciones aledañas a viviendas como graneros, talleres, almacenes, depósitos y garajes, que no estuvieran abandonados y fueran empleados diariamente por la población. Además, fueron elegidos sitios rurales cercanos a viviendas donde fueran practicadas actividades agrícolas o ganaderas. Todas estas áreas estuvieron ubicadas en un radio no mayor a 150 metros de viviendas habitadas.

Captura y procesamiento de roedores

Los roedores fueron capturados mediante el empleo de trampas tipo Sherman live-capture traps ( $8 \times 9 \times 23$ cm; Sherman Traps, Inc., Tallahassee, FL) en todas las áreas domésticas y peridomésticas identificadas como sitios de estudio. Se utilizó como cebo, avena humedecida con vainilla y mantequilla de maní. Fueron colocadas 50 trampas a una distancia no mayor de 5 metros en forma radial, durante 79 noches de trampeo. Las trampas fueron colocadas en la tarde y revisadas en la mañana siguiente. Los animales capturados fueron retirados de las trampas y procesados para la recolección de muestras de acuerdo a las normas estándares de bioseguridad $(7,8)$.

Básicamente, una vez capturados los roedores fueron transportados al Instituto de Investigaciones Biológicas del Trópico (IIBT) de la Universidad de Córdoba. Una vez en el laboratorio, cada animal fue anestesiado mediante el empleo de clorhidrato de ketamina al $10 \%$, y una muestra de sangre fue colectada mediante punción intracardíaca con una jeringa heparinizada. Las muestras sanguíneas obtenidas fueron centrifugadas a 3500 r.p.m durante 5 minutos. El suero obtenido se colocó en un baño serológico a $60{ }^{\circ} \mathrm{C}$ durante 30 minutos con el fin de inactivar los virus que pudieran estar presentes en la muestra. Estos se almacenaron en crioviales de $2 \mathrm{~mL}$ a $-70{ }^{\circ} \mathrm{C}$ hasta su análisis. Los animales previamente anestesiados fueron sacrificados por dislocación cervical y se registraron en una base de datos parámetros morfométricos como longitud total, medida de cola, patas y orejas, peso, sexo y estatus reproductivo. Los cráneos y la piel de los roedores capturados fueron utilizados para su clasificación taxonómica, además, de las lecturas de la carta dentaria descritas por el Museo Americano de Historia Natural $(9,10)$. Después del procesamiento, cada espécimen fue colocado directamente en un recipiente con etanol al $80 \%$ ó formalina al $10 \%$ por 3 días, antes de su inmersión en etanol al $70 \%$ para su conservación a largo plazo (6).

Análisis de las muestras por ELISA 
Las muestras sanguíneas de roedores conservadas en tiras de nobuto (30 mm x 20 mm x 13 mm; Toyo Roshi Kaisha, Ltd. Tokio, Japón) fueron enviadas al Special Pathogen Branch del Center for Diseases Control and Prevention, Atlanta, Georgia, USA, donde fueron analizadas para la detección de anticuerpos IgG, empleando como antígeno una proteína recombinante de la nucleocapside del Sin Nombre Virus (SNV) por el método de inmunoensayo enzimático-ELISA anteriormente descrito (11). Básicamente, muestras sanguíneas diluidas 1: 25 en $5 \%$ de leche descremada en 0,01 M de tampón-fosfato-salino con 0,5 Tween-20, fueron nuevamente diluidas de 1: 100 hasta 1:6400 en cuatro diluciones dobles en placas de microtitulación. Cada muestra se analizó contra la proteína recombinante de la nucleocapside y un antígeno control. Un conjugado de IgG anti-Rattus norvigicus y anti-Peromyscus leucopus (cadenas pesadas y ligeras) (Kirkegaard and Perry, Gaithersburg, MID) fue empleado para detectar la inmunoglobulina unida (11).

Análisis de la información

La información fue recolectada mediante un formulario estandarizado e incorporada a una base de datos. Para su análisis se usaron las herramientas de análisis de datos de la hoja de calculo de Excel 2000®. Para valorar diferencias entre sexos y estado reproductivo en cuanto a seroprevalencias, se utilizó la prueba de Ji cuadrado.

Aspectos éticos para la toma de muestras a partir de roedores

El Comité de Investigación del Instituto de Investigaciones Biológicas del Trópico de la Universidad de Córdoba aprobó el protocolo para la toma de muestra a partir de cada uno de los roedores capturados. Debido a que la obtención de la muestra sanguínea por punción intracardíaca y la obtención de los cráneos para la posterior clasificación taxonómica, llevaría a la muerte de todos los roedores; los animales previamente anestesiados con clorhidrato de ketamina al 10 \% se les practicó eutanasia por dislocación cervical.

\section{RESULTADOS}

\section{Roedores capturados}

En 79 noches de trampeo utilizando por cada noche 50 trampas tipo Sherman live-capture traps ( 8 x 9 x 23 cm; Sherman Traps, Inc., Tallahassee, FL), fueron capturados un total de 336 roedores durante los meses de Enero de 2003 a Noviembre de 2004 en once municipios del Departamento de Córdoba-Colombia (Figura 1). La captura de los roedores fue evaluada por el calculo de 
la densidad relativa, estimado como el número de capturas/total de trampasnoche x 100. Así, con un total de 3950 trampas-noche se obtuvo un éxito de captura del 8,5\%.

Los géneros de roedores capturados y su distribución por familia, sub-familia-género y reactividad para hantavirus en cada uno de los municipios del departamento de Córdoba, son mostrados en el Tabla 1.

Los roedores muridos fueron el grupo más común de roedores capturados, representando el 94,3 \% de la muestra. Del total de capturas, 74,1 \% (249/336) pertenecieron a la sub-familia Murinae; 19,9 \% (68/336) a la subfamilia Sigmodontinae. Solo el 5 \% (17/336) se incluyeron dentro de la familia Heteromyidae y 0,6 \% (2/336) en la familia Echimyidae. Cada una de las familias y sub-familias estuvieron representadas por los siguientes géneros: Murinae: Muss musculus (31,8 \%); Rattus rattus (26,2 \%); $R$. norvegicus (8,3 \%) y Rattus sp (7,7 \%). Sigmodontinae: Sigmodon (2,4 \%); Zygodontomys (0,6 \%); Oryzomys (14\%) y Oligoryzomys (3,3\%). El total de los roedores capturados de la familia Heteromyidae, estuvo representado solo por el género Heteromys (5,1 \%), al igual que la familia Echimyidae, por el género Proechimys (0,6 \%).

Figura 1. Distribución geográfica de la reactividad para hantavirus en el total de roedores capturados por municipio en el Departamento de Córdoba-Colombia

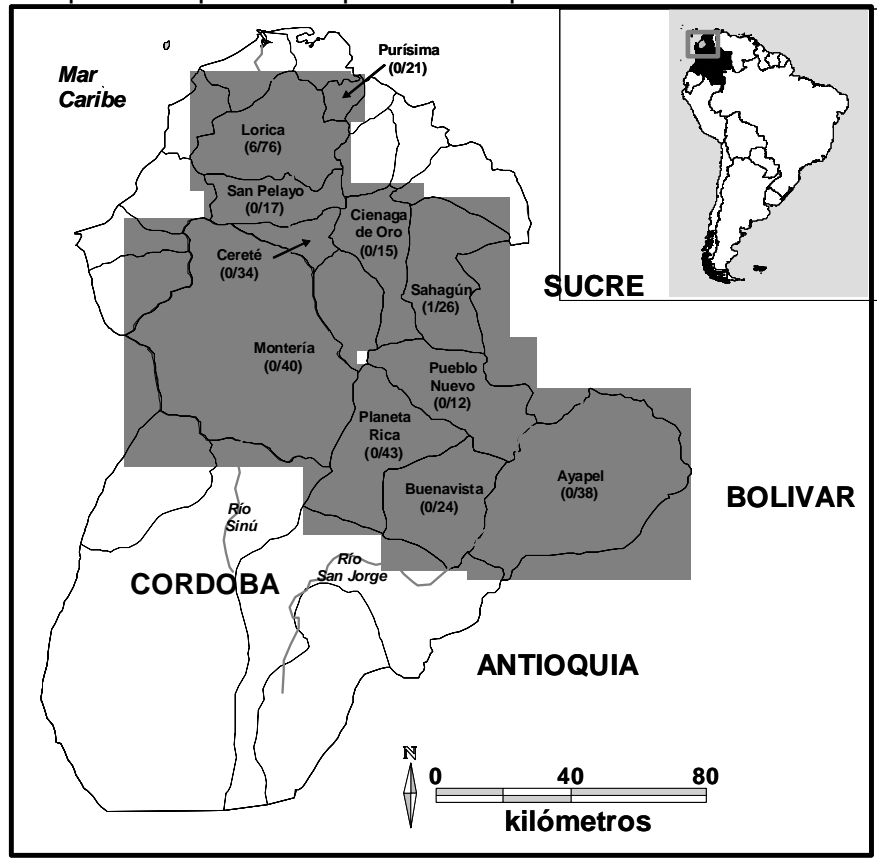


Tabla 1. Distribución de roedores capturados en los municipios del departamento de Córdoba-Colombia según sub-familia-género y reactividad para hantavirus

\begin{tabular}{|c|c|c|c|c|c|c|c|c|c|c|c|c|}
\hline \multirow[t]{2}{*}{$\begin{array}{l}\text { CLASIFICACIÓN } \\
\text { TAXONÓMICA }\end{array}$} & \multicolumn{11}{|c|}{$\begin{array}{l}\text { NÚMERO DE ROEDORES CAPTURADOS POR MUNICIPIOS DE ESTUDIO (No } \\
\text { SEROPOSITIVOS) }\end{array}$} & \multirow[b]{2}{*}{ TOTAL } \\
\hline & LOR & PR & MON & AYA & CER & $\mathrm{SAH}$ & BTA & PUR & $\mathrm{CO}$ & PVO & $\mathrm{PYO}$ & \\
\hline \multicolumn{13}{|l|}{$\begin{array}{l}\text { SUB-FAMILIA } \\
\text { MURINAE }\end{array}$} \\
\hline M. MUSCULUS & 24 & 5 & 14 & 24 & 1 & 0 & 17 & 0 & 10 & 8 & 4 & 107 \\
\hline R. RATTUS & 12 & 28 & 7 & 12 & 19 & 1 & 4 & 0 & 0 & 2 & 3 & 88 \\
\hline R. NORVEGICUS & 2 & 7 & 8 & 2 & 4 & 0 & 3 & 0 & 1 & 1 & 0 & 28 \\
\hline RATTUS SP & 0 & 3 & 11 & 0 & 9 & 0 & 0 & 0 & 3 & 0 & 0 & 26 \\
\hline \multicolumn{13}{|l|}{$\begin{array}{l}\text { SUB-FAMILIA } \\
\text { SIGMODONTINA } \\
\text { E }\end{array}$} \\
\hline S. HISPIDUS & 0 & 0 & 0 & 0 & 0 & 8 & 0 & 0 & 0 & 0 & 0 & 8 \\
\hline $\begin{array}{l}\text { ZYGODONTOMY } \\
\text { S }\end{array}$ & 0 & 0 & 0 & 0 & 1 & 0 & 0 & 0 & 1 & 0 & 0 & 2 \\
\hline ORYZOMYS & $30(4)$ & 0 & 0 & 0 & 0 & 0 & 0 & 17 & 0 & 0 & 0 & 47 \\
\hline OLIGORYSOMYS & $6(1)$ & 0 & 0 & 0 & 0 & 1 & 0 & 4 & 0 & 0 & 0 & 11 \\
\hline $\begin{array}{l}\text { FAMILIA } \\
\text { HETEROMIYDAE } \\
\text { HETEROMYS }\end{array}$ & 2(1) & 0 & 0 & 0 & 0 & 14 & 0 & 0 & 0 & 1 & 0 & 17 \\
\hline $\begin{array}{l}\text { FAMILIA } \\
\text { ECHIMYIDAE } \\
\text { PROECHIMYS }\end{array}$ & 0 & 0 & 0 & 0 & 0 & $2(1)$ & 0 & 0 & 0 & 0 & 0 & 2 \\
\hline TOTA!L & 76 & 43 & 40 & 38 & 34 & 26 & 24 & 21 & 15 & 12 & 7 & 336 \\
\hline
\end{tabular}

Exito de captura (número de capturas/total de trampas-noche x 100) = 8,5\%; Lorica (LOR), Planeta rica (PR), Montería (MON), Ayapel (AYA), Cereté (CER), Sahagún (SAH), Buena vista (BTA), Purísima (PUR), Cienaga de oro (CO), Pueblo Nuevo (PVO) y San Pelayo (PYO). 
El porcentaje de capturas realizadas durante el período de muestreo en el total de municipios oscilo entre el 2,1 \% (7/336) y el 22,6 \% (76/336), en San Pelayo y Lorica, respectivamente. Los géneros con un menor índice de capturas pertenecieron a la sub-familia Sigmodontinae. El 53,3 \% de los roedores fueron hembras en un estado reproductivo juvenil, solo el 41,9 \% (75/179) fueron adultos. Por el contrario, en el total de roedores machos $(n=157)$ el porcentaje de aislamientos según el estado reproductivo juvenil o adulto (juvenil: 50,9 \%; adulto: 49,1 \%) no presentó diferencias significativas $(\mathrm{p}>0.50)$.

\section{Prevalencia de anticuerpos}

La seroprevalencia de anticuerpos contra hantavirus en el total de roedores capturados fue del 2,1 \% (7/336). El mayor porcentaje de roedores seropositivos pertenecieron a la subfamilia Sigmodontinae (71,4 \%). Lorica y Sahagún fueron los únicos municipios donde se capturaron roedores seropositivos para hantavirus mostrando seroprevalencias del $1,8 \%$ y $0,3 \%$, respectivamente (Tabla 1).

\section{DISCUSIÓN}

En la actualidad, sur América constituye una de las principales zonas endémicas, donde se han registrado más de 1500 casos de SPH y se ha identificado la gran mayoría de los hantavirus existentes. Países como Costa Rica, Panamá, Venezuela, Perú, Brasil, Argentina, Uruguay, Paraguay, Bolivia y Chile, se han sumado al constante informe de casos de SPH y reporte de nuevos hantavirus y sus huéspedes reservorios $(3,12)$. En Colombia, aunque ningún caso de SPH clínicamente confirmado ha sido informado hasta el momento, una seroprevalencia de infección por hantavirus del 13,5 \% fue identificada en poblaciones humanas trabajadoras del campo (4). El reconocimiento del SPH ha incrementado el interés en la ecología de los huéspedes reservorios y una serie de estudios transversales y longitudinales fueron iniciados para describir la amplitud de la infección hantaviral, la ecología del reservorio y la dinámica virus-huésped en especies de reservorios de sur América. Como consecuencia de ello, estudios de roedores han permitido la identificación de reservorios putativos de hantavirus en Uruguay, Brasil, y Argentina, y detectado anticuerpos en algunas especies de roedores no previamente reportadas en Paraguay, Venezuela y Panamá (3).

La tasa de seroprevalencia del 2,1 \% identificada en este estudio, contrasta con los reportes serológicos para hantavirus descritos en sur América, los 
cuales han mostrado tasas más elevadas (3-7 \%) (13-15). Estos estudios incluyeron solo roedores sigmodontinos y la seropositividad se incrementó entre el $3 \%$ y el $11 \%(13,14,16-18)$. En nuestro estudio, cuando se consideró solo roedores de esta sub-familia, la tasa se incrementó a 7,35 \%. Una explicación probable a la baja prevalencia obtenida en Colombia comparada con tasas de infección de roedores reportadas en países como Argentina, Chile y Panamá, podría ser la alta densidad de roedores sigmodontinos reconocidos en estos países. De hecho, algunas variantes genéticas (19-21) o cepas de hantavirus han sido descritas en Argentina y Panamá en más de 2 especies de roedores sigmodontinos.

Este es el primer estudio encaminado a estudiar la circulación de hantavirus en huéspedes roedores en el caribe colombiano. La seroprevalencia identificada hasta el momento en estas poblaciones de roedores reservorios, probablemente se deba a que el mayor porcentaje de roedores capturados $(74,1$ \%) pertenecieron a la sub-familia Murinae (ratas y roedores del viejo mundo); población de roedores en la cual no se identificó ningún reservorio positivo para hantavirus al igual que lo descrito por Pini et al. en el 2003 (22).

Aunque el propósito del estudio no era establecer diferencias entre los géneros en cuanto a seropositividad, los porcentajes de positividad para hantavirus específicos por género, oscilaron entre 5,9 \% (1/17) y $50 \%$ (1/2), para Heteromys y Proechimys, respectivamente. Esta variabilidad pudo ser distinta de contarse con un tamaño mayor de especimenes. Sin embargo, teniendo en cuenta estos resultados, es posible que roedores muridos pertenecientes a la sub-familia Murinae, no estén actuando como reservorios principales y/o huéspedes competentes para hantavirus en la región caribe colombiana y que otros géneros de roedores del neotrópico como Heteromys sp y Proechimys sp, cuyos reportes de seropositividad para hantavirus aún son escasos en sur América (23), podrían comportarse como spillover de este virus emergente.

Por otra parte, el grupo de roedores sigmodontinos, los cuales ya han sido identificados como reservorios putativos de hantavirus en sur América, estuvieron representados por un 20,2 \% del total de capturas realizadas. Solo los géneros Oligoryzomys (1/336) y Oryzomys (4/336), fueron positivos para hantavirus, presentando seroprevalencias de 0,3\% y 1,2 \% sobre el total de roedores sigmodontinos capturados, respectivamente. Sumado a esto, todos los roedores sigmodontinos positivos (5/7) fueron juveniles (machos: $n=3$, hembras: $n=2$ ). Además, la seropositividad general encontrada según su estado reproductivo (1,7 \% hembras y $2,6 \%$ en machos) no presento diferencias significativas $(\mathrm{p}=0.56)$. Estos resultados contrastan con seroprevalencias 
previamente reportadas para hantavirus en roedores sigmodontinos las cuales superan el 10\% (24). Sin embargo, apoyan la premisa que las altas poblaciones de roedores jóvenes que estuvieron presentes durante el momento del muestreo, podrían haber generado un fenómeno de dilución del virus, debido al constante recambio de la población que involucraría una población adulta mucho más reducida. Esto condicionaría una menor naturaleza competitiva de los machos adultos durante la época de apareamiento y la búsqueda de alimentos y, por lo tanto una menor frecuencia de contacto entre machos adultos y machos jóvenes.

En conclusión, la prevalencia de anticuerpos contra el SNV en roedores de Córdoba, Colombia; indica que al menos un hantavirus es endémico en roedores del norte colombiano y estaría frecuentemente trasmitido a residentes rurales, previamente identificados como poblaciones de alto riesgo de contagio, por estudios de seroprevalencia reportados en este país (4). Además, este hallazgo apoya la evidencia que los hantavirus y el SPH continúan siendo un problema de salud pública panamericano, en especial para países en vía de desarrollo como Colombia. Estudios posteriores, encaminados a intensificar el número de capturas tanto en áreas no exploradas como en sitios donde ya ha sido detectada seropositividad en seres humanos, serían necesarios para incrementar el conocimiento sobre la epidemiología del SPH y resolver cuales son los hantavirus que están circulando en Colombia y así establecer su relación filogenética con otros hantavirus prevalentes en Sur América y el mundo •

Agradecimientos. Este trabajo fue desarrollado durante el programa de jóvenes investigadores e innovadores del Instituto Colombiano para el Desarrollo de la Ciencia y la Tecnología "Francisco José de Caldas"-COLCIENCIAS-2005. Agradecemos al Dr. Nelson Alvis, Ph.D., de la Universidad de Cartagena, por su gran aporte en el análisis estadístico de los datos. Igualmente, reconocemos toda la colaboración del Dr. Kent Wagoner, Ph.D., del Special Pathogens Branch, Division of Viral and Rickettsial Diseases, Centers for Disease Control and Prevention-CDC, Atlanta, USA., en la elaboración del mapa georeferencial de la reactividad de hantavirus en roedores capturados en el Departamento de Córdoba.

\section{REFERENCIAS}

1. Schmaljohn C, Hjelle B. Hantaviruses: a global disease problem. Emerg Infect Dis 1997; 3(2):95-104 
2. Organización Panamericana de la Salud (OPS). Hantavirus en las Américas: guías para el diagnóstico, el tratamiento, la prevención y el control. Epidemiología de la enfermedad en los humanos. Cuadernos técnicos de la OPS, $N^{\circ} 47$. OPS, Washington, DC; 1999.

3. Pini N. Hantavirus pulmonary syndrome in Latin America. Current Opinion Infect Dis 2004; 17(5):427-31.

4. Mattar S, Parra M. Serologic evidence of Hantavirus infection in Human, Colombia. Emerg Infect Dis 2004; 10(12):2263-2264.

5. Eisenberg JF. Mammals of the neotropics: the northern neotropics. Panama, Colombia, Venezuela, Guyana, Suriname, French Guiana. Chicago: University of Chicago Press, 1989; vol.1:329.

6. Salazar-Bravo J, Armien B, Suzán G, Armien A, Ruedas LA, Avila M, et al. Serosurvey of Wild Rodents for Hantaviruses in Panamá, 2000-2002. J Wildlife Dis. 2004; 40(1):103-109.

7. Mills J, Childs J, Ksiasek T, Peters CJ, Velleca W. Methods for trapping and sampling small mammals for virologic testing. U.S. Deparment of Health \& Human services. Public Health Service. Centers for Disease Control and Prevention. Atlanta, Georgia 30333; 1995.

8. Mills J, Yates T, Childs J, Parmeter R, Ksiasek T, Rollin P, et al. Guidelines for working with rodents potentially infected with Hantavirus. J Mammalogy 1995; 76(3):716-722.

9. Carleton MD, Musser GG. Systematic Studies of Oryzomine Rodents (muridae, Sigmodontinae): A Synopsis of MicrOryzomys. Bulletin of the American Museum of Natural History 1989; 191.

10. Voss RS. Mammalian Diversity in Neotropical Lowland Rainforests: A Preliminary Assessment1996; 230.

11. Feldmann H, Sanchez A, Morzunov S, Spiropoulou CF, Rollin PE, Ksiazek TG, et al. Utilization of autopsy RNA for the synthesis thesis of the nucleocapsid antigen of a newly recognized virus associated with hantavirus pulmonary syndrome. Virus Res 1993; 30(3):351-67.

12. Organización Panamericana de la Salud (OPS). [Internet] Ministerios de Salud de los países respectivos. Prevención y Control de Enfermedades/Enfermedades Transmisibles/ Enfermedades Emergentes y Reemergentes. Números de casos de y defunciones por Síndrome Pulmonar por Hantavirus (SPH) (Región de las Américas, 1993-2004). Disponible en Internet en: http://www.paho.org/Spanish/AD/ DPC/CD/hantavirus-1993-2004.htm. Consultado en Agosto 25 de 2005.

13. Pavletic C. Hantavirus: Su distribución geográfica entre los roedores silvestres de Chile. Rev Chil Infect 2000; 17(3):186-196.

14. Calderón G, Pini N, Bolpe J, Levis S, Mills J, Segura E, et at. Hantavirus reservoir host associated with peridomestic habitats in Argentina. Emerg Infect Dis 1999; 5(6):792-797.

15. Cantoni G, Padula P, Calderon G, Mills J, Herrero E, Sandoval P, et al. Seasonal variation in prevalence of antibody to hantaviruses in rodents from southern Argentina. Trop Med Int Health 2001; 6(10):811-816. 
16. Yahnke CJ, Meserve PL, Ksiazek TG, Mills JN. Patterns of infection with Laguna Negra virus in wild populations of Calomys laucha in the central Paraguayan chaco. Am J Trop Med Hyg 2001; 65(6): 768-776.

17. Williams RJ, Bryan RT, Mills JN, Palma RE, Vera I, Velásquez F, et al. An outbreak of hantavirus pulmonary syndrome in western Paraguay. Am J Trop Med Hyg 1997; 57(3): 274-282.

18. Toro J, Vega JD, Khan AS, Mills JN, Padula P, Terry W, et al. An outbreak of hantavirus pulmonary syndrome, Chile, 1997. Emerg Infect Dis 1998; 4(4): 687-694.

19. Padula PJ, Colavecchia SB, Martínez VP, Gonzales MO, Eldelstein A, Miguel $\mathrm{DL}$, et al. Genetic diversity, distribution, and serological features of hantavirus infection in five countries in South America. J Clin Microbiol 2000; 38(8):3029-3035.

20. Padula PJ, Sánchez AJ, Edelstein A, Nichol ST. Complete nucleotide sequence of the M RNA segment of Andes virus and analysis of the variability of the termini of the virus S, M and L RNA segments. J Gen Virol 2002; 83(9):2117-2122.

21. Bohlman MC, Morzunov SP, Meissner J, Beth-Taylor M, Ishibashi K, Rowe J, et al. Analysis of hantavirus genetic diversity in Argentina: S segment- derived phylogeny. J Virol 2002; 76(8):3765-3773.

22. Pini N, Levis S, Calderón G, Ramírez J, Bravo D, Lozano E, Ripoll C, St. Jeor S, Ksiazek T, Barquez R, Enria D. Hantavirus Infection in Humans and Rodents, Northwestern Argentina. Emerg Infect Dis 2003; 9:1070-1076.

23. Rosa E, Mills J, Padula P, Elkhoury M, Ksiazek T, Mendes W, Santos E, Araújo G, Martinez V, Rosa J, Edelstein A, Vasconcelos P. Newly Recognized Hantaviruses Associated with Hantavirus Pulmonary Syndrome in Northern Brazil: Partial Genetic Characterization of Viruses and Serologic Implication of Likely Reservoirs. Vector Borne Zoonotic Dis 2005; 5: 11-19.

24. Torres F, Navarrete J, Aldunate R, Yates T, Mertz G Vial P, Ferrés M, Marquet P, Palma R. Peridomestic small mammals associated with confirmed cases of human hantavirus disease in southcentral Chile. Am J Trop Med Hyg 2004; 70: 305-309. 
